\title{
BALLISTOCARDIOGRAM. II. NORMAL STANDARDS, ABNORMALITIES COMMONLY FOUND IN DISEASES OF THE HEART AND CIRCULATION, AND THEIR SIGNIFICANCE ${ }^{1}$
}

\author{
BY ISAAC STARR AND HENRY A. SCHROEDER \\ (From the Department of Research Therapeutics, the Department of Pharmacology, and the \\ Medical Division of the Hospital of the University of Pennsylvania, Philadelphia)
}

(Received for publication November 18, 1939)

The Ballistocardiograph (1), a modern adaptation of an old idea, consists of a table suspended from the ceiling on wires and braced to prevent motion in any but the longitudinal direction. Motion in this direction, opposed by a strong steel spring, is magnified about 8,000 times and photographed.

When a subject lies on the table he is not conscious of its motion but the records obtained are characteristic and reproducible. Figures 1, 3, and 4 give typical examples. The chief forces producing this motion have been identified as the recoil from the ejection of blood from the heart, the impact of the blood striking the arch of the aorta and the curve of the pulmonary artery and, of less importance, the recoil from the systemic blood accelerated feetward when the aortic arch has been passed. The resultant of these forces is not perfectly reproduced in the record, the chief difficulty being due to the physical properties of body tissues which vibrate for a brief instant after receiving a single blow. These aftervibrations warp the descending part of the record but they have a much smaller effect on the ascending part, and from this part reliable data can be secured.

Evidence has been presented (1) that the size of the initial waves, $I$ and $J$, is related to the cardiac output and that the form of the ballistic curve is determined by the shape of the curve of blood velocity in the great vessels. These conceptions lead one to expect that, when the circulation is feeble, the ballistocardiogram will be of low amplitude and, when the heart is weak, the form of the ballistic record will be altered.

Therefore, we believe that this simple method will permit the easy identification of patients with

1 The completion of this work was assisted by a grant from the Penrose Fund of the American Philosophical Society. abnormal circulations and will also provide evidence of cardiac health or disease of a type which has no counterpart in the ordinary clinical tests.

To realize this expectation it was first necessary to determine the normal standards. To this end over 200 healthy persons from 20 to 84 years of age were examined and standards of normality have been obtained from the results. Ballistocardiograms have also been secured on over 400 patients and this experience has permitted description and analysis of the more common variations from the normal.

\section{TECH NIQUE}

At first all tests were performed with the subject under conditions of basal metabolic rate, but we soon abandoned this in favor of a test made after 15 or 30 minutes' rest and not less than 2 hours after a meal. The chief reason for this change was convenience but we justified it by several arguments. It is true that the basal ballistocardiogram is of somewhat lower amplitude than that obtained in the resting condition described, but the differences found in pathological conditions are so much larger that confusion seems most unlikely. One can point to an analogy with the blood pressure. The basal blood pressure is considerably lower than the value obtained at other times but the differences found in disease are so large that to restrict one's attention to basal values is unnecessary for most purposes.

In another respect our technique changed early in the investigation. We started with the belief that the results would be more comparable if the weight on the table was kept constant and, accordingly, added iron bars until the combined weight of subject and bars was $200 \mathrm{lbs}$. After working 3 months with this plan it was abandoned because of the discovery that the additional dead weight diminished the damping and so increased the error due to the secondary vibrations. For the heavier men this error was negligible, but for the lightest women the extra weight increased the sum of the altitudes of waves $I$ and $J$ by almost 20 per cent. For the following 6 months all subjects were tested both with and without the extra weight and from this experience a correction was established. In this report the values obtained on normal subjects during the first 3 months have been corrected to 
conform to the larger data obtained during the last 3 years, without extra weighting.

Our standard technique of taking records is as follows: The subject lies supine on the table with his heels in firm contact with the foot plate. He is allowed one or two pillows. After a rest period of $\mathbf{1 5}$ minutes (longer if exercise has recently been taken), the operator assures himself that the subject is quiet and relaxed, and photographs the table's motion. The record should extend through several respiratory cycles; more is not necessary for most purposes.

In addition, we usually secure records with each patient holding the breath in both the inspiratory and expiratory positions with the glottis open.

Reading the Records. The record is first inspected for artifacts due to the subject's failure to lie still. Such artifacts often distort the cardiac complexes and they can be identified because the distorted form is never exactly repeated. Only the forms which are regularly repeated deserve attention. Vibrations in the building are of such high frequency that they cause no confusion, though they may blur the margins of the record.

The effect of respiration is next assessed. When breathing is normal no diaphragmatic impacts appear on the record, but the amplitude of the cardiac complexes varies with the phases of respiration. If this variation is regular, it is sufficient to study two complexes selected as typical of the highest and the lowest of the respiratory cycles.

These typical complexes are measured. After selecting a base line by inspection, the altitude of waves $H, I$, and $J$ are measured to the nearest $1 / 2 \mathrm{~mm}$. The duration of waves $I$ and $J$ are measured along the base line in fractions of seconds.

The effect of one wave upon another, the aftervibrations, may be discovered by setting calipers at a distance which corresponds to 0.17 seconds, the average vibration period of human bodies (1), and ascertaining the time relation of the peaks. After having identified aftervibrations and their effects, one can identify and describe abnormalities of form and estimate the cardiac output.

Calculation of cardiac output. Our ballistocardiogram is calibrated so that a static force of $\mathbf{2 8 0}$ grams displaces the light spot image $1 \mathrm{~cm}$. Our formulae for estimating cardiac output apply only to an instrument with this calibration. If used for another instrument, a correction inversely proportional to the difference in calibrations must be employed. Thus, if 280 grams displace the image 2 $\mathrm{cm}$, the estimations of cardiac output from our formulae must be halved.

When the ballistocardiogram has a normal form and the respiratory variations are regular, cardiac output per minute may be estimated from measurements of two typical complexes by one of two methods. In most of the results reported in this paper we proceeded as follows :

Having ascertained that waves $H, I$, and $J$ were in phase with the body's period, as they almost always are, the altitude of $I$ was corrected by subtracting from it 40 per cent of the altitude of $H$; and $J$ by subtracting
TABLE I

Constants for estimating aortic internal diameter from age and body surface derived from Basett's data (2)

\begin{tabular}{|c|c|c|c|c|c|c|c|c|}
\hline \multirow{2}{*}{ Age } & \multicolumn{2}{|c|}{ Constants } & \multirow{2}{*}{ Age } & \multicolumn{2}{|c|}{ Constants } & \multirow{2}{*}{ Age } & \multicolumn{2}{|c|}{ Constants } \\
\hline & $a$ & $b$ & & $a$ & $b$ & & $a$ & $b$ \\
\hline $\begin{array}{l}20 \\
22 \\
24 \\
26 \\
28 \\
30 \\
32 \\
34 \\
36 \\
38\end{array}$ & $\begin{array}{l}1.2 \\
1.3 \\
1.4 \\
1.4 \\
1.5 \\
1.6 \\
1.6 \\
1.7 \\
1.8 \\
1.9\end{array}$ & $\begin{array}{l}0.1 \\
0.1 \\
0.1 \\
0.2 \\
0.2 \\
0.2 \\
0.2 \\
0.2 \\
0.3 \\
0.3\end{array}$ & $\begin{array}{l}40 \\
42 \\
44 \\
46 \\
48 \\
50 \\
52 \\
54 \\
56 \\
58\end{array}$ & $\begin{array}{l}2.0 \\
2.1 \\
2.1 \\
2.2 \\
2.3 \\
2.4 \\
2.5 \\
2.6 \\
2.7 \\
2.8\end{array}$ & $\begin{array}{l}0.3 \\
0.3 \\
0.4 \\
0.4 \\
0.5 \\
0.6 \\
0.7 \\
0.8 \\
0.8 \\
0.9\end{array}$ & $\begin{array}{l}60 \\
62 \\
64 \\
66 \\
68 \\
70\end{array}$ & $\begin{array}{l}3.0 \\
3.1 \\
3.2 \\
3.3 \\
3.4 \\
3.6\end{array}$ & $\begin{array}{l}1.0 \\
1.1 \\
1.3 \\
1.4 \\
1.6 \\
1.8\end{array}$ \\
\hline
\end{tabular}

When $S=$ body surface area in sq. meters. Aortic internal diameter in $\mathrm{cm} .{ }^{2}=a S-b$.

40 per cent of $I$. The corrected values of $I$ and $J$ for the two typical complexes were then averaged.

From the subject's surface area and age the aortic cross section, $A$, was estimated from Bazett's data (2) which are reproduced in a more convenient form in Table I, or read directly from his Figure 3 (2).

The duration of the cycle, $C$, was estimated in seconds by dividing the pulse rate per minute into 60 . For convenience, values of $C^{a / 2}$ for various pulse rates are given in Table II. The values found were entered in the fol-

TABLE II

Algebraic functions of the cardiac cycle in terms of heart rate

\begin{tabular}{|c|c|c|c|c|c|c|c|c|}
\hline Pulse & $C^{2 / 3}$ & $\sqrt{C}$ & Pulse & $C^{2 / 2}$ & $\sqrt{C}$ & Pulse & $C^{2 / 2}$ & $\sqrt{C}$ \\
\hline $\begin{array}{c}\text { per } \\
\text { nisurute } \\
50 \\
52 \\
54 \\
56 \\
58 \\
60 \\
62 \\
64 \\
66 \\
68\end{array}$ & $\begin{array}{l}\text { sec- } \\
\text { onds } \\
1.32 \\
1.24 \\
1.18 \\
1.06 \\
1.03 \\
1.00 \\
0.96 \\
0.91 \\
0.87 . \\
0.83\end{array}$ & $\begin{array}{l}\text { sec- } \\
\text { onds } \\
1.09 \\
1.07 \\
1.05 \\
1.03 \\
1.02 \\
1.00 \\
0.98 \\
0.97 \\
0.95 \\
0.94\end{array}$ & $\begin{array}{c}\text { per } \\
\text { minute } \\
70 \\
72 \\
74 \\
76 \\
78 \\
80 \\
82 \\
84 \\
86 \\
88\end{array}$ & $\begin{array}{l}\text { sec- } \\
\text { onds } \\
0.80 \\
0.76 \\
0.73 \\
0.70 \\
0.68 \\
0.65 \\
0.63 \\
0.61 \\
0.59 \\
0.57\end{array}$ & $\begin{array}{l}\text { sec- } \\
\text { onds } \\
0.92 \\
0.91 \\
0.90 \\
0.89 \\
0.88 \\
0.86 \\
0.85 \\
0.84 \\
0.83 \\
0.83\end{array}$ & $\begin{array}{c}\text { per } \\
\text { minute } \\
90 \\
92 \\
94 \\
96 \\
98 \\
100 \\
102 \\
104 \\
106 \\
108\end{array}$ & $\begin{array}{l}\text { sec. } \\
\text { onds } \\
0.55 \\
0.53 \\
0.51 \\
0.49 \\
0.48 \\
0.47 \\
0.45 \\
0.44 \\
0.43 \\
0.42\end{array}$ & $\begin{array}{l}\text { sec- } \\
\text { onds } \\
0.82 \\
0.81 \\
0.80 \\
0.79 \\
0.78 \\
0.77 \\
0.76 \\
0.76 \\
0.75 \\
0.75\end{array}$ \\
\hline
\end{tabular}

lowing formula (1) which was solved with the help of a slide rule:

$$
\text { Average cardiac stroke volume }=7 \sqrt{(3 I+2 J) A C^{3 / 2}} \text {, }
$$

the answer being in cc.

For example, subject S, age 43, is $6 \mathrm{ft}$. tall, weighs 185 lbs. The pulse rate is 75 . In a typical large complex $H=3, I=7, J=10 \mathrm{~mm}$, and in a typical small complex $H_{2}=2, I_{2}=4, J_{2}=5 \mathrm{~mm}$.

Correcting the wave altitudes

$$
\begin{aligned}
& I_{\text {cor }}=7-(3 \times 0.40)=5.8 \\
& J_{\text {cor }}=10-(7 \times 0.40)=7.2 \\
& I_{2 \operatorname{cor}}=4-(2 \times 0.40)=3.2 \\
& J_{2 \operatorname{cor}}=5-(4 \times 0.40)=3.4 .
\end{aligned}
$$


And averaging $I_{\mathrm{av}}=4.5$ and $J_{\mathrm{av}}=5.3$.

The subject's surface area is 2.05 square meters. From Table I we find, $a=2.1$ and $b=0.35$. The internal aortic area, $A=2.1(2.05)-(0.35)=3.96 \mathrm{~cm}^{2}$.

For a pulse rate of $75, C^{3 / 2}$ is found from Table II to be 0.72 seconds.

Substituting these values in equation (1) we obtain:

Average stroke volume

and $\quad=7 \sqrt{[3(4.5)+2(5.3)](3.96)(0.72)}=58 \mathrm{cc}$.

Cardiac output $=\frac{0.058 \times 75}{2.05}$

$=2.1$ liters per minute per sq. meter body surface

or

Cardiac output $=\frac{58 \times 75}{185}$

$=23$ cc. per minute per lb. body weight.

The cardiac output can be calculated in another manner which is superior in certain respects to that just outlined, for it is mathematically sounder and it avoids the corrections. To apply this method the areas within waves $I$ and $J$ must be estimated. As these waves are nearly triangular and one can construct a triangle whose area approximates the area of the wave to be measured, the base of each triangle is measured in fractions of a second, the altitude in $\mathrm{mm}$. Therefore, the area under wave $I=\int I d t=1 / 2$ (base $X$ altitude) and the area under wave $J$ has a similar formula.

One may then use the following formula (1), finding $A$ as before and $\sqrt{C}$ from the pulse rate in Table II.

Stroke volume of any complex in cc.

$$
=33 \sqrt{\left(2 \int I d t+\int J d t\right) A \sqrt{C}} \text {. }
$$

Both formulae for cardiac output give very similar results. We started by preferring the altitude formula as simpler but have gradually come to prefer the area method.

\section{Normal standards}

Selection of healthy subjects. Two hundred healthy persons were secured from the faculty and students of the medical school, the staff and attendants of the hospital, and the friends and relatives of the authors. The group as a whole had sedentary occupations and no one was regularly engaged in hard physical labor. The age ranged from 20 to 84 years. All considered themselves in good health and were doing work commensurate with their age. Many had had physical examinations within the last few years and their intelligence and cooperativeness were such that we feel sure they suppressed no information at their command. We included a few who had not always been healthy. Thus 3 had once had pulmonary tuberculosis but it had been

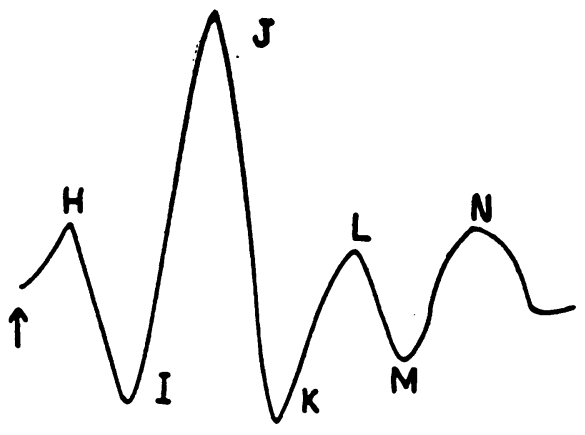

Fig. 1. Diagram of the Normal Ballistocardiogram with the Letters UsEd to Designate the Waves

The arrow indicates the position of the beginning of the QRS. complex of the electrocardiogram. Reproduced by permission of the Am. J. Physiol.

arrested for many years. Certain other former illnesses will be mentioned also.

The decision as to whether to accept the older subjects as normal was not always easy. We first decided to exclude all whose systolic blood pressure exceeded $150 \mathrm{~mm}$. $\mathrm{Hg}$ or whose diastolic pressure exceeded $90 \mathrm{~mm}$., but we encountered very few elderly women who could pass the test. Since Lewis (3) found that the average blood pressure of his series of healthy men increased rapidly between the ages of 62 and 85 , we decided to admit any one over 60 whose pressure was below 170/100.

\section{Standards for cardiac output}

Our aim is ultimately to find the standards most useful in detecting disease. These must be established by prolonged experience. To make a start, we fell back on the old conception of the uniformity of nature, believing that the arrangement of our data which made the normal group most homogeneous was the most likely to be useful. To avoid the inclusion of unrecognized cases of cardiac disease, the greatest emphasis has been placed on the data obtained on subjects under 40 years of age given in Table III.

The results have been recorded in Figure 2 in the form of frequency diagrams. Cardiac output per minute per sq. meter of body surface was studied first. The grouping of the data around the means of the younger groups was most satisfactory, but we were surprised at the difference between the mean values of men and women in the second and third decades; a difference not statis- 
tically significant (for $\mathrm{P}=0.05$ ), but certainly not negligible.

As an explanation of this sex difference, it occurred to us that a goodly number of the young

TABLE III

Statistics concerning the cardiac output of healthy young men and women

\begin{tabular}{|c|c|c|c|c|c|c|c|c|}
\hline \multirow[t]{2}{*}{ Sex } & \multirow[t]{2}{*}{ Age } & \multirow[t]{2}{*}{$\operatorname{Num}_{\text {ber }}$} & \multicolumn{2}{|c|}{$\begin{array}{c}\text { Cardiac out- } \\
\text { put liters per } \\
\text { minute per } \\
\text { sq. meter } \\
\text { body surface }\end{array}$} & \multicolumn{2}{|c|}{$\begin{array}{c}\text { Cardiac out- } \\
\text { put cc. per } \\
\text { minute per } \\
\text { lb. body } \\
\text { weight }\end{array}$} & \multicolumn{2}{|c|}{$\begin{array}{c}\text { Cardiac out- } \\
\text { put cc. per } \\
\text { minute per } \\
\text { lb. ideal } \\
\text { weight }\end{array}$} \\
\hline & & & Mean & $\sigma$ & Mean & $\sigma$ & Mean & - \\
\hline $\begin{array}{l}M \\
F \\
M \\
F\end{array}$ & $\begin{array}{l}20-29 \\
20-29 \\
30-39 \\
30-39\end{array}$ & $\begin{array}{l}27 \\
30 \\
31 \\
18\end{array}$ & $\begin{array}{l}\text { liters } \\
2.06 \\
1.75 \\
2.04 \\
1.87\end{array}$ & $\begin{array}{r}\text { per } \\
\text { cent of } \\
\text { mean } \\
9.5 \\
12.0 \\
11.3 \\
8.0\end{array}$ & \begin{tabular}{|c|}
$c c$. \\
24.2 \\
22.8 \\
23.6 \\
23.2
\end{tabular} & \begin{tabular}{|c} 
per \\
cent of \\
mean \\
11.7 \\
13.5 \\
12.3 \\
10.1
\end{tabular} & $\begin{array}{c}c c . \\
26.4 \\
22.4 \\
25.0 \\
23.2\end{array}$ & 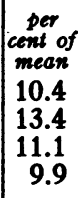 \\
\hline Average & & & 1.91 & 10.2 & 23.4 & 11.9 & 24.2 & 11.2 \\
\hline
\end{tabular}
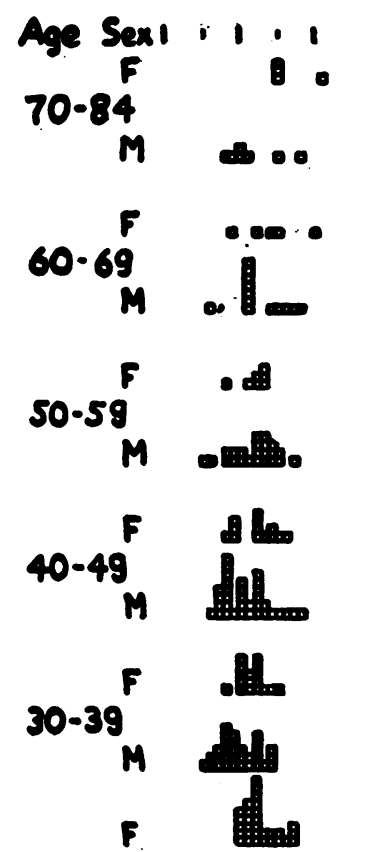

$20-29$

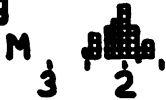

liters per min.

per sq. meter body surfoce

Fig. 2. Frequency Diagram

Cardiac output per minute of healthy persons in terms of body surface, body weight, and ideal weight, for both sexes in the adult decades. The vertical dotted lines define the normal limits which we usually employ. women were too thin, having kept their weight down for the sake of their appearance. Loss of weight does not affect the surface area proportionately so we estimated the cardiac output per minute in terms of the body weight, the results being given in Figure 2. In these data the difference between men and women is largely eliminated but the scattering is a little greater than in the preceding series.

Searching for causes of this scattering, we found that the lowest values were frequently obtained on subjects definitely overweight. Fatty tissue has an extremely small blood supply so that the weight of such subjects might be a poor index of their circulatory needs. Therefore, we estimated the cardiac output per minute in terms of " ideal weight," a figure based on height and age (4). In these data the difference between the mean values in young men and young women reappeared and the scattering was improved very little.

Inspection of the data of Figure 2 discloses a tendency for the averages to diminish with age, as was found by Lewis (7) and by Starr et al (5); and this is especially true when ideal weight is employed. But the differences are small, again as in Lewis' data, and they might be due to the accidental inclusion of elderly persons who were not truly normal. Therefore, realizing that further experience may cause us to change our policy, at present we prefer to use the normal limits found in the younger persons for the older ones as well. If anyone prefers to employ standards based on body surface or ideal weight, then separate standards for men and women should be employed and the normal limits may be derived from the data given in Figure 2 and Table III, the boundaries being twice the standard deviation above and below the mean. At present we prefer to use the body weight and this is discussed below.

\section{Studies of simpler methods as indices of cardiac output}

Before the cardiac output standards were compiled, we had planned to identify abnormal cases in some simpler way. We had investigated the possibility of employing the ballistic amplitude alone as an index of stroke volume and defining normal standards on this basis. After measuring the altitude of $I+J$ in $\mathrm{mm}$. for representa- 
tive large and small complexes, we made the following calculation for each case in the normal series:

Amplitude index $=\frac{\left(I+J+I_{2}+J_{2}\right) \times \text { Pulse rate }}{\text { body weight }}$.

The results showed that normal ballistic amplitude decreased with age, making it necessary to have a different standard for each decade. Also, averages of the two sexes differed, and the results had double the standard deviations and were scattered over twice the range of the corresponding data of cardiac output.

We also tried another index:

"Square Root of Amplitude" index

$$
=\frac{\sqrt{I+J+I_{2}+J_{2}} \times \text { Pulse rate }}{\text { body weight }} \text {. }
$$

These results were but little better than the preceding. No shortcut has been found which gives results on normal subjects as uniform as those obtained when the cardiac output is calculated.

Limits of normality. To detect abnormal circulations we employ standards based on cardiac output per beat per pound of body weight, as shown in Figure 2. The normal range has been chosen so that the chances are about 97.5 in 100 that any values falling either above or below are abnormal. From the data given in Table III we calculate a normal range of from 29 to $18 \mathrm{cc}$. per minute per lb. Applying this limit to the 149 apparently healthy persons under 50 , we find that 8 of them gave subnormal values. Two of these were very plump young women in the third decade who were 43 and $15 \mathrm{lbs}$. over their ideal weights; the single man in the fourth decade was extremely obese, weighing $275 \mathrm{lbs}$; of the 3 men in the fifth decade, 1 had once been suspected of hypothyroidism, another was an arrested case of pulmonary tuberculosis who took no exercise, the third was $40 \mathrm{lbs}$. over his ideal weight. Therefore, one could adopt the view that this limit should not be applied to persons overweight and use the data based on ideal weight for such, or one could maintain that many obese persons have abnormal circulations. As they have one symptom characteristic of the subnormal circulation (8), i.e. undue dyspnea on exertion, the latter view seems more logical.

Inspection of Figure 2 shows that the great majority of healthy persons over 50 years of age have maintained their circulations within the normal range. Of those who are subnormal, possible explanations may be cited for several; one man in the sixth decade had recovered from an attack of exophthalmic goiter about 10 years before ; another had had "myocarditis" diagnosed in the army in 1917. But in most of the others no explanation was obvious although we received the impression that they were less active than their confreres. Whether the normal limit should be lowered in the older group can hardly be decided without more experience.

Therefore, a lower normal limit of $18 \mathrm{cc}$. per minute per $1 \mathrm{~b}$. body weight seems a reasonable starting point in the search for a useful division between normal and subnormal circulations.

\section{Standards for the normal form of the ballistocardiogram}

In Figures 3 and 4 are records obtained on 8 normal persons chosen to illustrate the extent of the normal variation. We have obtained records similar to these in over 600 individuals.

The normal form can be recognized at a glance. $H I$ is always sharp and clearly defined. $H I$ and $I J$ make acute angles with the vertical and they occur in the first half of systole. The $J$ peak dominates the record.

Normal variations in systole. The prominent variation in amplitude accompanies the respiratory cycle and it is consistent with well known physiological conceptions. During inspiration the descent of the diaphragm steadily increases the pressure in the abdomen while it diminishes that in the thorax; therefore, as the pressure gradient in the veins becomes greater, cardiac filling increases and output increases also. During expiration the reverse takes place. This respiratory variation is always conspicuous on normal records if the breath is not held; its absence may well be of pathological significance.

The $K$ wave, mostly an artifact (1), varies more than the other prominent waves.

Normal variations in diastole. Waves $L$ and $M$ are prominent in most normal subjects (Figure $3, A$ ), much less prominent in a few (Figure $3, B)$. At the end of diastole there is a ripple in some records and $H$ varies with its relation to 


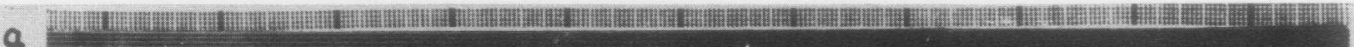

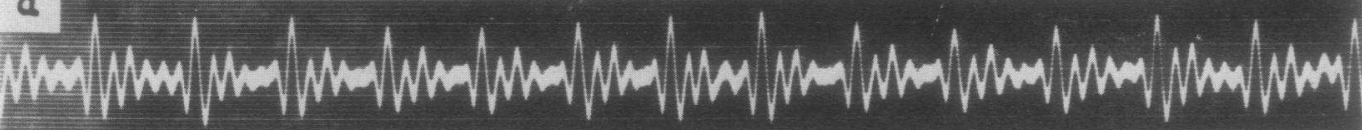

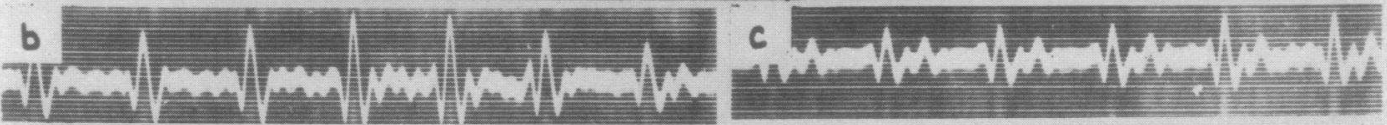

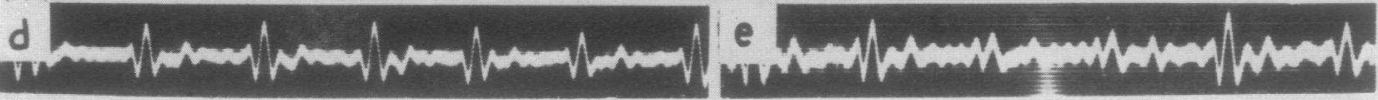

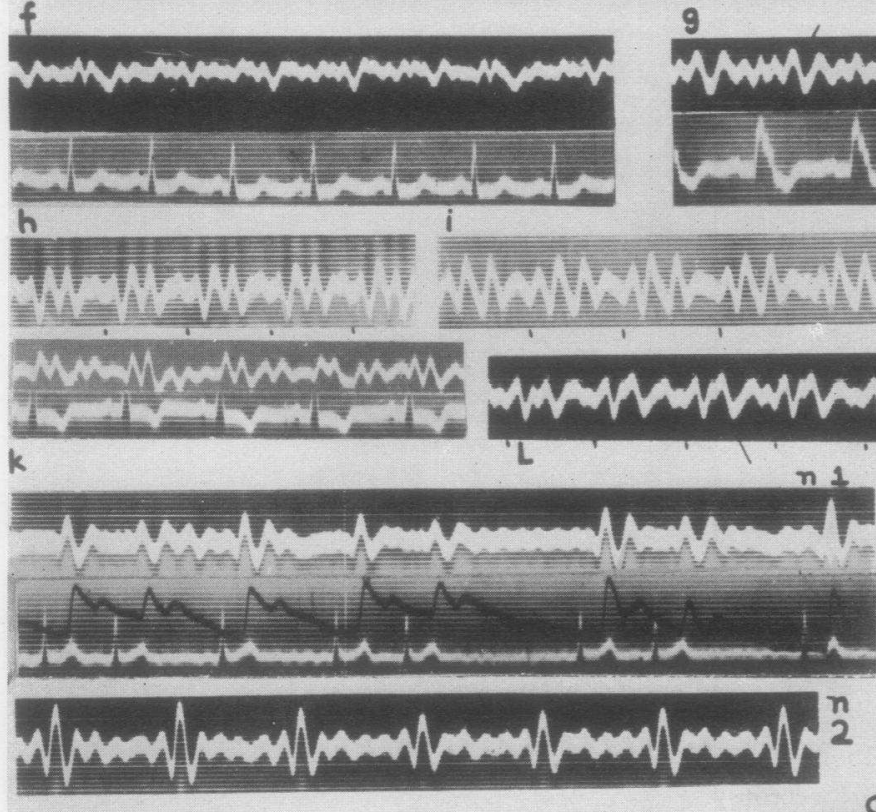

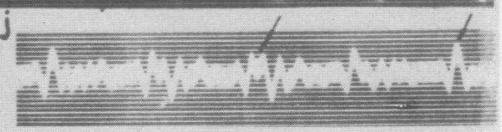

m

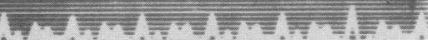

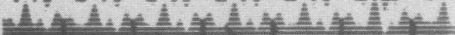

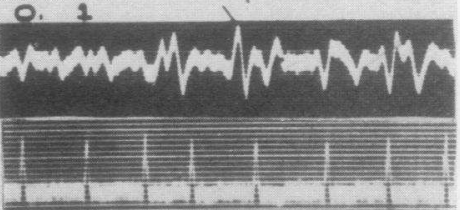

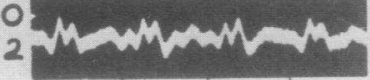

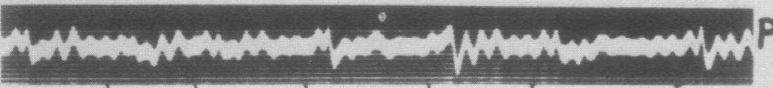

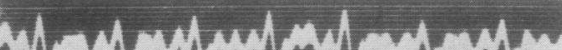
R. I

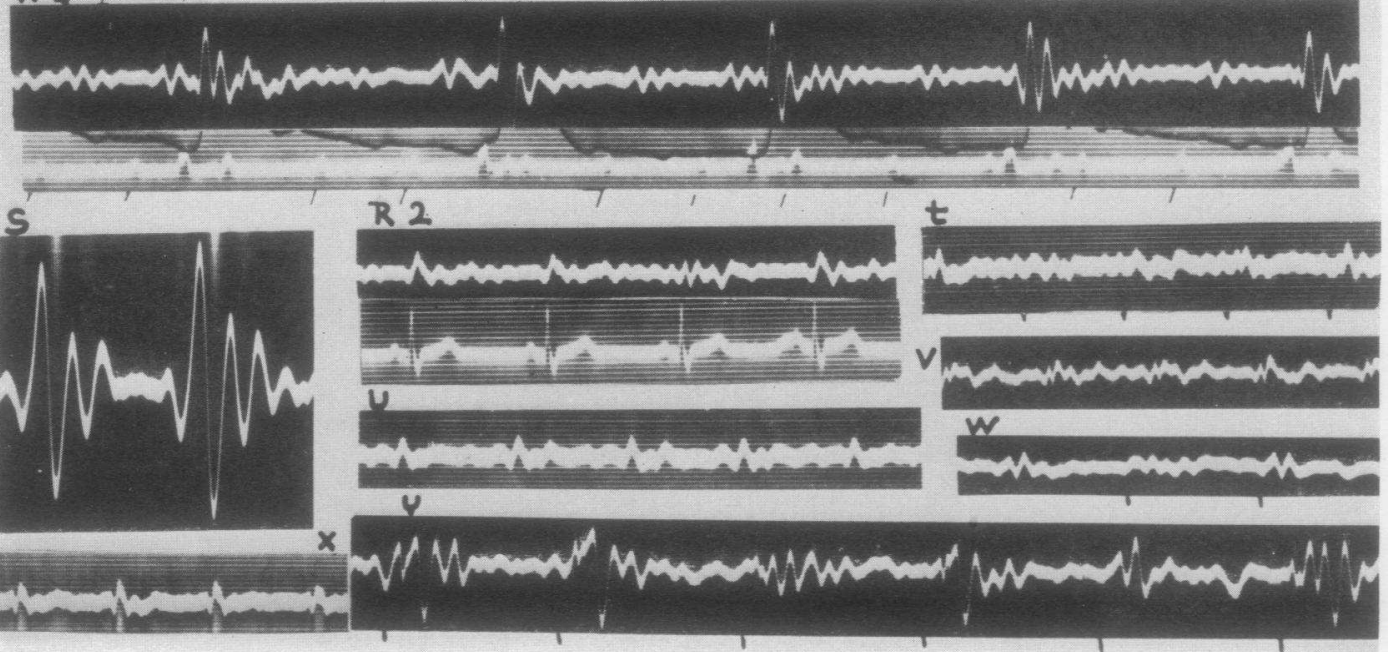

FIG. 3. 
these vibrations. In other records the end of diastole is flat. These differences are artifacts (1). When delivered in phase, the systolic impacts start the body vibrating, but when the last impact is out of phase no after-vibrations appear. No sig-

Fig. 3. Normal and Abnormal Bali.istocardiograms Rediced to $1 / 2$ Actual Size

The time record on the top applies to all records but S. The vertical coordinates are the same for all records; the upward direction indicates a movement headward. Horizontal lines on or under records indicate the beginning of the QRS. complex in a simultaneous electrocardiogram. Diagonal lines point to features of interest. Venous pressures, except when indicated below, were well within normal limits.

$A$; J. D.; male, age 33 , height $5 \mathrm{ft} .7$ inches, weight 145 lbs., BP. $120 / 70 \mathrm{~mm}$. $\mathrm{Hg}$, diagnosis normal.

$B$; F. J.; m., 23, 5 ft. 11, 152, 134/70, normal.

$C$; E. C.; f., 44, 5 ft., 124, 100/65, normal.

$D$; C. E.; m., 28,5 ft. $8,135,105 / 70$, normal.

$E$; E. L.; m., 64, 5 ft. 10, 145, 130/70, normal. (A vibration in the building blurs the first part of the record.)

$F$; H. F.; m., 46, 5 ft., 146, 170/110. Hypertensive cardiovascular disease. Simultaneous EKG., lead 1. Note low amplitude, shallow $I$ wave, low $J$, prominent downstroke late in systole; "late downstroke type."

$G$; C. H.; m., 56, $6 \mathrm{ft}$., 170, 100/65. Bundle branch block. Necropsy 2 months later showed old infarct at apex of left ventricle. Note "late downstroke," shallow $I$, in left hand part, a "late $M$ " on right.

$H$; B. S. ; m., 56,5 ft. 6, 170, 180/130, venous pressure 20. Lues, hypertension, in congestive failure; necropsy 3 months later, massive infarct of left ventricle. Note "late $M$ " shape; the first upward limb of the $M$ is the $J$ wave.

$I$; I. C.; m., 36, 5 ft. 8, 122, 120/90. Coronary occlusion 2 weeks before, recovered. Note "late $M$ 's."

$J$; C. M.; m., 64, 5 ft. 11, 141, 170/105. Severe angina pectoris. Note split $J$ peaks confined to part of respiratory cycle.

$K$ : J. R. ; m., 56, 5 ft. 3, 131, 186/110. Essential hypertension, EKG. suggests severe myocardial damage. Note "carly M's"; the first limb is the $H$ wave.

$L$; L. B. ; m., 45, 5 ft. 5, 127, 120/80. Hypernephroma? EKG. left bundle branch block. Note doubling of $J$ peak.

$M$; M. S.; f., 23, 5 ft. $10,123,102 / 70$. Rheumatic heart disease. Mitral stenosis Class II $B$. EKG. shows extremely high $\mathrm{P}$ waves. Note flattening of $I$ wave valley.

N.1; A. B. ; m., 39, 5 ft. 6, 148, 120/80. Rheumatic heart disease. Class II $A$. Mitral stenosis, auricular fibrillation. EKG. and brachial pulse. Note diversity of form in ballistic record, largest amplitude after the pauses.

N.2 ; same patient 4 days later. Blood pressure $140 / 85$, normal sinus rhythm after quinidine. Note increased amplitude. nificance should be attached to differences of this kind.

\section{Description of abnormal forms}

Four hundred patients have been studied in the search for abnormalities. The great majority had

O.1; M. B.; m., 67, 5 ft. 8, 197, 160/110. Hypertensive cardiovascular disease, auricular fibrillation. Note ballistic complexes of diverse form; the large deflection in the center is probably an artifact.

0.2 ; same patient 7 days later after spontaneous resumption of normal sinus rhythm. Blood pressure 160/ 105. Note "late" $M$-shaped tracings.

$P$; W. L. ; m., 55, 5 ft. 3, 160, 128/85. Arterial heart disease, former coronary thrombosis, auricular fibrillation. Note low amplitude and diversity of ballistic form.

$Q$; C. G.; f., 48, 5 ft. 3, 188, 205/128. Arteriolonephrosclerosis. Note combination of late $M$ and late downstroke type of ballistocardiogram.

R.1; W. P.; m., 58, 5 ft. 1, 126, 120/70. EKG. and bottom of brachial pulse record. Auriculoventricular block. Note effect of isolated auricular contractions on ballistic record and variability of the ventricular complexes.

R.2; Same subject reverted to normal sinus rhythm. Note the surprising contrast with the previous record.

$S$; J. F.; m., 24, 5 ft. 10, 128, 166/25. Rheumatic heart disease, wide open aortic regurgitation with all peripheral signs. Cor bovinum. Note huge amplitude and normal form. In this picture the film ran more rapidly than in the others. Distance for 1 second on scale at top equals 0.67 seconds for this record. The vertical scale is identical with that of all other records in this figure.

$T$; P. M.; f., 58, 4 ft. 8, 125, 210/100. Arteriolonephrosclerosis. Gall bladder disease? EKG. normal. Heart size normal. Note extremely small ballistic amplitude.

$U$; B. H.; f., 49, 5 ft. 2, 159, 145/90. Angina pectoris, myxedema. Basal metabolic rate-39 per cent. Given thyroid; collapsed on street 2 months after test. Necropsy, old infarct of anterior wall of left ventricle and of interventricular septum, coronary sclerosis.

$V$; M. L.; f., 48, 5 ft. 9, 129, 115/70. Neurocirculatory asthenia with attacks of paroxysmal tachycardia, now normal sinus rhythm. Note low amplitude. $J$ peak doubled in some complexes.

$W$; P. H.; m., 58, 5 ft. 6, 152, 150/90. Diabetes mellitus. Angina pectoris for many years; severe pain, possibly a fresh infarct 3 days before test; recovered. Note low ballistic amplitude, form normal.

$X$; A. C.; f., 55, 5 ft., 138, 160/90. Diabetes mellitus. Coronary occlusion 2 years before, angina since, died in an attack 12 months later. No necropsy. Note low amplitude.

$Y$; H. R.; m., 46, 5 ft. 7, 180, 126/74. Convalescent pneumonia, bradycardia, ventricular escape. Myocardial infarct? Record obtained after giving paredrine. Note extraordinary diversity of form of the systolic complexes. 


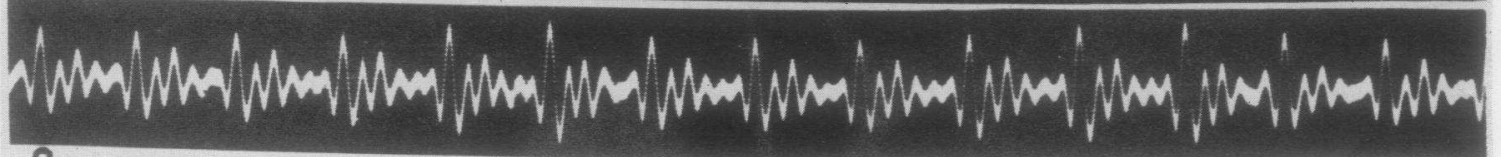
a

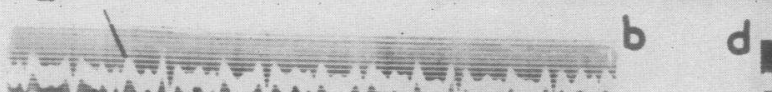

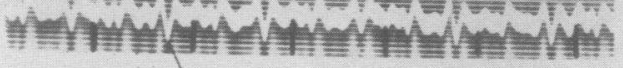

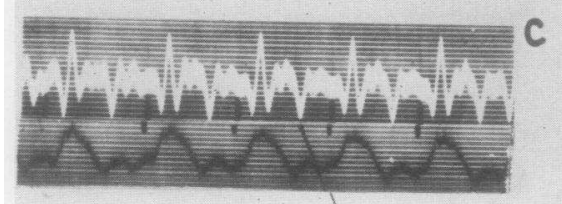

d 2

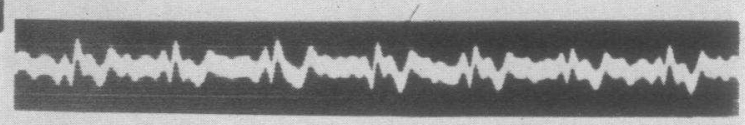

.

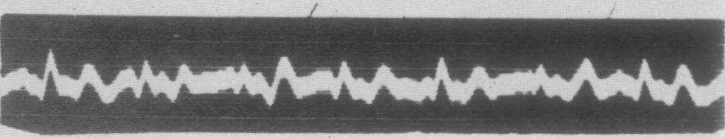

e 1

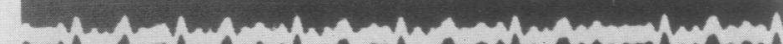

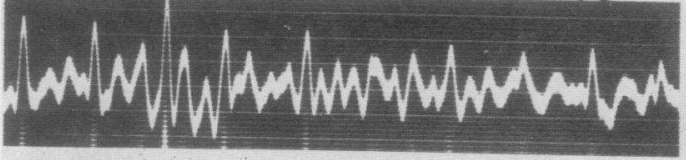

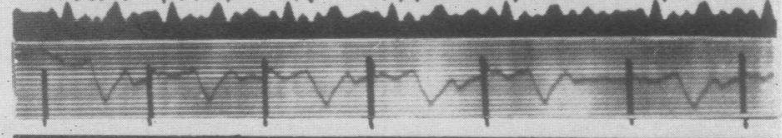

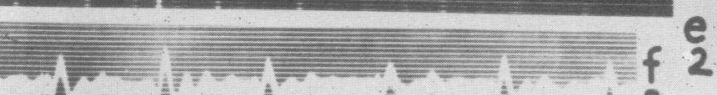

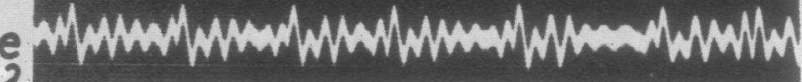

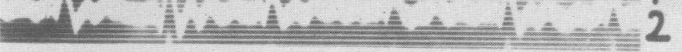

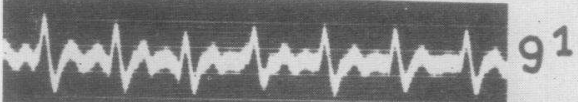

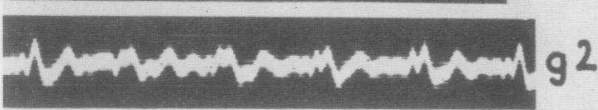
i1 1 $|\mathrm{m} / \mathrm{m} / \mathrm{m} / \mathrm{mumm} / \mathrm{mm} / \mathrm{mm}| \mathrm{|}$

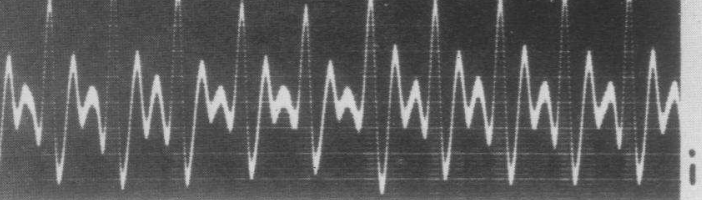

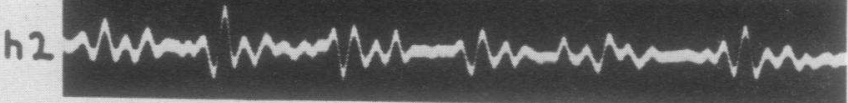
j 1

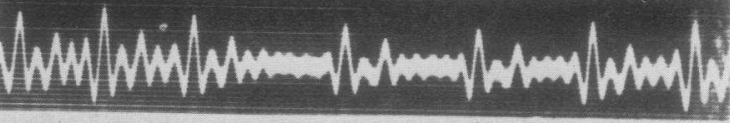

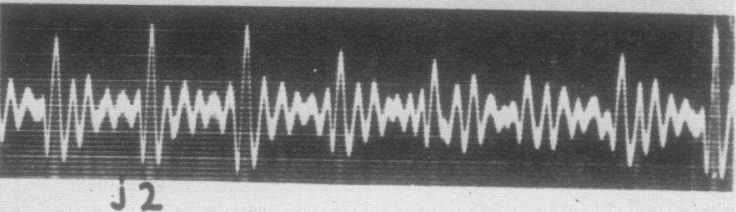
2

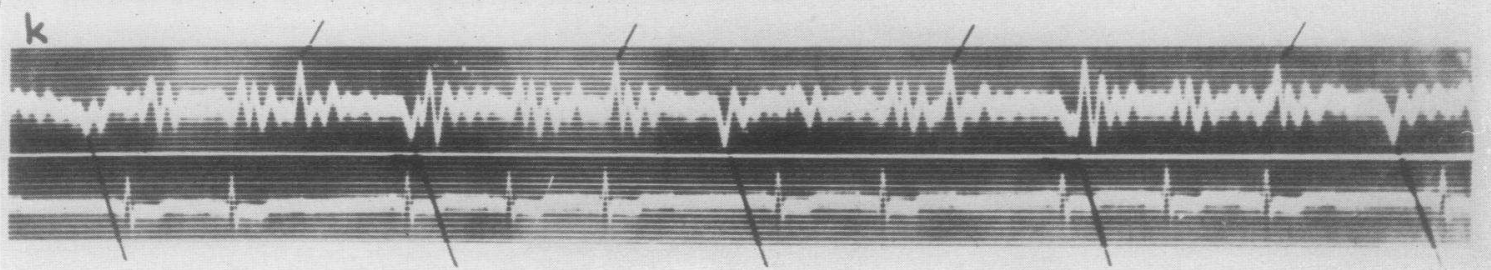

FIG. 4. 
records in the normal forms. When abnormalities were encountered, ballistocardiograms and electrocardiograms were recorded simultaneously on the same film. ${ }^{2}$ In certain instances, records of carotid or brachial pulses obtained by glycerine tambours and Frank capsules were made simultaneously. In other cases the jugular venous pulse was recorded. The ballistic abnormalities encountered may be classified as follows:

The late downstroke type. This is characterized by a shallow and indefinite $I$ wave, followed by a slow, often fluctuating, rise to $J$, terminated by a sharp downstroke, $J K$, occurring late in systole and making the most prominent feature of the record. Evidence has been presented (1) that ballistocardiograms of this type are due to abnormal curves of blood velocity in the great vessels, the maximum expulsion velocity not being attained until late in systole. Changes of this type have been produced experimentally in dogs by asphyxia (1).

2 We are indebted to Dr. C. C. Wolferth of the Robinette Foundation for the loan of an electrocardiograph for this purpose.

Fig. 4. Normal and Abnormal Ballistocardiograms 1/2 Actual Size

The time record at the top applies to all. Vertical black lines on or below the records indicate the position of the beginning of the QRS. complex in a simultaneous electrocardiogram. Diagonal lines point to important features of the records. Venous pressures were normal except when given below.

$A$; A. R.; age 40 , height $5 \mathrm{ft} .9$ inches, weight 145 lbs., B.P. $130 / 75$, diagnosis normal.

$B$; L. W.; 63, 5 ft. 9, 132, 100/70. Arteriosclerotic heart disease. Right bundle branch conduction defect. Note diastolic impacts greater than systolic.

$C$; M. G.; 49, 5 ft. 6, 131, 100/75. Venous pressure $15 \mathrm{~cm}$. $\mathrm{H}_{2} \mathrm{O}$. Rheumatic heart disease, mitral stenosis, tricuspid regurgitation with pulsating liver. Record with jugular pulse. Note constant diastolic downward wave in a record otherwise normal.

D.1; M. vB.; 35, 5 ft. 2, 165, 198/130. Essential hypertension. Note notch on $j k$ at certain phases of respiration.

D.2; Same, one week later, on thiocyanate. Blood pressure 130/90. Diastolic wave much more prominent.

E.1; K. R.; 37, 5 ft. 11, 144, 105/80. Venous pressure 22. Constrictive pericarditis, auricular fibrillation, ascites, record with jugular pulse. Note low systolic amplitude with well-marked diastolic waves.

E.2; Same, 2 months post operative. Blood pressure $105 / 65$. Venous pressure 10. Patient greatly improved
Figure 3, $F$ and $G$, shows examples of this type of abnormality in patients. We have encountered it in 12 subjects. Of these, 7 had hypertension with marked cardiac enlargement, 1 dying within a year. Another patient (Figure 3, $G$ ) had suffered from a coronary occlusion 2 years before which was demonstrated at necropsy 2 months later. Another patient (Figure 4, G.1), a diabetic, showed the abnormality only when she was in severe acidosis. In contrast to these obviously sick people, the same abnormality was found in a young woman with atypical angina attacks who gave no objective evidence of heart disease, and again in a man of 52 who considered himself healthy but admitted marked dyspnea on exertion. The experience at hand suggests that this abnormality indicates serious myocardial dysfunction.

The "late $M$ " shaped type. In this type of record the first peak of the $M$ corresponds to the $J$ wave of the normal record. The form can be explained by the assumption that one side of the heart is strong, the other weak; the stronger, ejecting blood in a normal manner, produces maximum velocity early in systole, while the weaker, ejecting

but still had auricular fibrillation; note increase in amplitude.

F.1; J. E.; 38, 5 ft. 71/2, 163, 148/70. Hyperthyroid. Basal metabolic rate +35 per cent. The patient could not keep still and her movements confuse the record, although the systolic complexes can be clearly seen.

F.2 ; same patient 2 months after partial thyroidectomy. Blood pressure $130 / 85$. Basal metabolic rate -7 per cent. A striking contrast.

G.1; E. D.; 58, 5 ft. 1, 120, 132/74. Diabetes mellitus coming out of severe acidosis, flushed and weak. Note shallow or suppressed $I$ wave, late downstroke type.

G.2; same, 11 days later. Blood pressure 145/90. No acidosis now. Note marked decrease in amplitude, lower complexes of " early $M$ " type in part of respiratory cycle.

H.1; T. R.; 60,5 ft. $8,142,96 / 70$. Venous pressure 5.5 After severe hemorrhage from hemorrhoids.

H.2 ; same, 1 hour later, after transfusion of $600 \mathrm{cc}$. blood. Blood pressure 100/75. Venous pressure 7.0. Note increase in amplitude.

I.1 and 2; J. B.; 22, 5 ft. 10, 133. Normal. Before and during inhalation of amyl nitrite.

J.1; E. C. ; 24,5 ft. 11, 145, 114/78. Normal, sinus arrhythmia.

J.2; Same, 10 minutes after $0.7 \mathrm{cc}$. adrenalin S.C. Blood pressure $146 / 78$.

$K$; M. F.; 64, 5 ft. 4, 129, 160/90. Arterial heart disease, irregular heart block. The diagonal lines on the record point to the effects of the diaphragmatic impacts caused by abnormally violent breathing. 
with difficulty, does not produce the maximum velocity until late in systole. Thus ballistic asynchronism implies neither asynchronism of the time of the cardiac systoles nor inequality of the output from the two hearts. This abnormality has been produced in animal experiments by damaging one side of the heart (1).

Figure 3, H, I, J, and $O .2$, shows typical examples of this type of abnormality in patients. We have obtained such records in 19 patients. Eleven were cases of old or recent coronary infarction; 3 were proved later at necropsy. Four were cases of hypertension without evidence of cardiac infarction; 1 came to necropsy. In 3 other cases the chief diagnoses were arteriosclerosis and anemia, luetic heart disease, and hyperthyroidism. The last case, a man aged 63 , had the abnormality without being acutely ill; his future course will be watched with interest. Four of the 19 cases died within a year, and experience indicates that this abnormality is of serious import.

The "early $M$ " type. This type (Figure 3, $K$ ) has a superficial resemblance to the preceding but the first limb of the $M$ is an elevated $H$ wave, not the $J$ wave. It can usually be distinguished by the absence of the conspicuous $H I$ downstroke before the $M$ in the "early" type. The normal $H$ wave is to be attributed to movement of the heart's mass during the pre-ejection phase of cardiac contraction. Exaggerated $H$ waves making " early $M$ " records have been most frequently recorded from cases of hypertension. In such instances it seems probable that the heart, struggling to eject blood against the increased resistance, moved so as to give a larger impact than the normal. But we have also seen this abnormality in a few patients without hypertension. One of these was a patient with a recent coronary occlusion who subsequently recovered. With this exception none of the patients showing the abnormality were very sick and it does not seem as serious as those discussed before.

Intermediate forms. The "late $M$ "-shaped curves as in Figure 3, $H$ and $I$, represent the extremes of ballistic asynchronism of the two ventricles and it is to be expected that all gradations will be found. Our theoretical conceptions (1) permit the arrangement of the records in a sequence. Small degrees of asynchronism would be expected to manifest themselves as a notch or shoulder on
$J K$, as shown in Figure 4, D.1; or as a flattening or doubling of the $J$ peak (Figure 3, L). Larger degrees would appear as $M$ 's whose central downward limb extends further and further down (Figure 3, $J$ and $O .2$ ), until finally, in the fully developed late $M$, the central limb crosses the base line (Figure 3, $H$ and $I$ ). It is to be expected that records of these kinds will indicate increasing degrees of the same pathological process.

Similarly, the late downstroke type represents the extreme condition in which the blood velocity attains its maximum very late in systole and gradations from the normal to this extreme are to be expected. If both ventricles impart to the blood a maximum velocity in mid systole, the theoretical ballistic curve is characterized by a doubling of the $I$ wave and a retardation of $J$. Records of this type are rare in our experience but the flattened. slightly notched $I$ and the retarded $J$ found in a case of rheumatic mitral stenosis (Figure 3, $M$ ) deserve this interpretation. A shallow $I$ wave, $H I$ having an abnormally obtuse angle with the vertical, is probably the first conspicuous sign of this abnormality. Figure 4, G.1 shows a record where the $I$ wave is very inconspicuous.

Abnormal diastolic waves. Preceded by a systolic complex of normal size, the normal diastolic wave is distorted by the after-vibrations. But its presence can be clearly recognized in most normal records since it causes an increase in the aftervibrations. Exceptions to this statement, such as Figure 3, $B$, are rare.

In some pathological conditions the systolic complexes are reduced in size and the diastolic waves become the most prominent feature of the record. Such records have been obtained most frequently when the venous pressure was elevated.

These abnormal diastolic waves are of two types: a sharp complex with maximum impact feetward, as shown in Figure $4, B$ and $C$, by the sharp downward deflection never seen in normal records; and a more rounded upward wave, as shown in Figure 4, D.2 and E.1, which resembles the normal diastolic wave but, disproportionately large, dwarfs the reduced systolic complexes, especially during expiration.

It is to be presumed that these diastolic impacts are set up by the rush of blood filling the heart and it is of interest that differences in the manner of filling may be detected in our ballistic records. 
Six of the 8 records showing diastolic abnormalities were obtained from patients who were seriously ill with advanced heart disease. In 1 patient with hypertension (Figure 4, D.2) the abnormality was not constant. The last patient, who had hypertension and diabetes, did not appear to be acutely ill.

Variations of form from beat to beat. In most records the characteristic form is maintained in every systole. The exceptions to this rule fall into two types.

In the first, the changes conform to the respiratory cycle. Figure $4, J$ illustrates this type; $M$ shaped complexes and intermediate forms alternate with normal complexes in each respiratory cycle. Apparently, when well filled, this heart is capable of discharging blood in a normal manner; when filling pressure is lowered the abnormality appears. This type of record has always been obtained from patients whose lives were in no immediate danger but it may well indicate the beginning of a pathological process.

In the second type, the changes of form have no relation to respiration. In auricular fibrillation the ballistic complexes vary greatly both in amplitude and in form. Often, as in Figure 3, $N .1$, the changes in amplitude are plainly due to the variations in rate, the heart expelling more blood after the pauses which permit greater filling. But, in addition to this, as shown in Figure 3, 0.1 and $P$, complexes of various sizes and types may follow each other indiscriminately in auricular fibrillation, giving impressive evidence of the disordered cardiac action. In all other conditions in which the electrocardiograms have varied from beat to beat, the impacts have also varied from beat to beat, sometimes producing most bizarre records, as is shown in Figure 3, $Y$. Extrasystoles may also produce ballistic records which vary much in form and amplitude.

The same beat-to-beat variation is found in complete heart block. Here the differences should be attributed in part to the confusion resulting when the auricular and ventricular impacts are superimposed, in part to variations in ventricular expulsion, depending on the unequal contributions made by the auricle towards its filling. The fact that the isolated auricular beats show so clearly on the ballistic records (Figure 3, R.1) is a tribute to the sensitivity of our apparatus. In the 4 cases of complete block tested, auricular deflections have always appeared on the ballistic record.

\section{DISCUSSION}

In attempting to ascertain the clinical utility of the ballistic records, two courses were open to us. We first planned to proceed empirically, describing the characteristics of the records obtained on healthy persons, recording the deviations from the normal encountered in disease, and attempting to explain them by the collateral evidence bearing on the case, such as the autopsy findings, $x$-ray appearance, after-histories, etc. This is the chief method by which clinical knowledge of the electrocardiogram has been advanced. But a second approach was available to us because the ballistic record, unlike the electrical record, is directly related to the heart's sole function, the pumping of blood. Therefore, we studied the relationship between the ballistic record and the cardiac output, a new and difficult field in which we regard our calculations as giving, at the best, only a crude approximation of the true situation (1). Nevertheless, from our mathematical solution we developed a formula for cardiac output which gave results superior to the data derived from measurements of the records, when both were subjected to the empirical test, correlation with results obtained by another cardiac output method (1). In short, by means of the theoretical approach we were able to build a better empirical method.

The results recorded in this paper show that we have repeated this experience for, by calculating cardiac output, even though our method is crude, we obtained a far more compact and logical set of normal standards than a simple measurement of the records provided. Therefore, as the physiological approach appears more promising than the purely empirical, we plan to report our results in physiological terms, as far as possible.

\section{Limitations of the method}

Tremors. Tics and gross tremors may ruin the record entirely. In a few very toxic patients with hyperthyroidism the uncontrollable trembling has rendered the record useless; in many such cases there is enough tremor to distort the cardiac complexes, as is shown in Figure 4, F.1. Emotional 
trembling may also cause trouble, but it yields readily to reassurance and attention to the patient's comfort. Good relaxation on the part of the subject is necessary to secure the best records.

Effects of abnormal respiration. If the subject's breathing is sufficiently vigorous, the impacts due to the respiratory movements are so much greater than the cardiac complexes that the latter cannot be identified. In some of these patients a satisfactory record can be obtained by having them hold their breath or at least breathe less violently for a brief period. In the worst cases sufficient reduction of breathing can usually be secured after a short period of voluntary hyperpnea.

Unfortunately, if the breath is held, the size of the ballistic complexes usually varies with the position of the diaphragm, being large if it is down, much smaller if it is up. Compression of air in the lungs, if the patient closes his glottis and performs the Valsalva experiment, also influences the ballistic amplitude profoundly. Under such circumstances, one must hesitate to draw conclusions concerning the cardiac output during natural breathing from records obtained when the breath is held or respiration voluntarily reduced. However, the form of the record has its usual significance and rough estimates of cardiac output are often possible. To study severe congestive heart failure, special difficulties must be overcome.

If abnormal breathing is not vigorous enough to destroy the record, the respiratory movements may produce characteristic impacts which appear on the record and confuse the cardiac complexes. These are shown diagrammatically in Figure $\mathbf{5}$ and as they appear on a record in Figure $4, K$. When present they must be identified or interpretation will suffer.

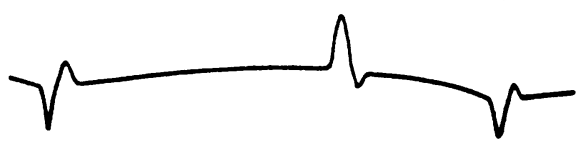

Fig. 5. Diagram of the Effect of Abnormally Violent Breathing on the Ballistic Record

Note arching of the base line and impacts when the respiratory movements change their direction.

Analysis of confused records. Occasionally, with diaphragmatic impacts present, with systolic complexes varying from beat to beat, with small indefinite systolic waves and diastolic waves of equal or greater amplitude, some records may appear on first glance as confusion worse con- founded. In such, interpretation is usually possible after comparison with records obtained when the breath is held with the diaphragm both up and down. Very occasionally additional evidence of the timing of systole, as by a simultaneous electrocardiogram, or an arterial pulse record, may be necessary to interpret the findings. These confused records have been found only in cases of heart disease of the most serious type and so we always regard them as of serious import to the patient.

Limitations in estimating cardiac output. The formulae given on page 438 apply only to records in the normal form. In such cases there is ample evidence that they have the rough accuracy characteristic of the other cardiac output methods. For the abnormal ballistic forms other formulae must be employed (1) and the evidence for their accuracy is still meager. Therefore, when the form is abnormal, we seldom attempt to express the cardiac output in quantitative terms. But recalling that, in the extremes of abnormality, the cardiac output may be about 35 per cent larger than the amplitude suggests (1), we do not hesitate to express an opinion on its size in most cases. Often the amplitude is so low that a glance reveals that the cardiac output was abnormal.

The cardiac output formulae contain an estimate of the internal cross section of the aorta from the subject's age and body surface, and errors in this estimate must be expected in certain pathological conditions. Such an error makes a smaller difference than one might suppose. If the aorta or the pulmonary artery actually had an internal section area which was twice that calculated, the estimated cardiac output would be only about 17 per cent too large. Abnormal aortic dilitation can be detected by $x$-ray examination. If the subject's weight is abnormal, ideal weight should be used in this estimation.

The cardiac output formulae also contain an estimate of the duration of ejection from the pulse rate and this holds only for normal rhythm. It would be easy to derive a formula for cardiac output in which the duration of systole was estimated in a different way, as from the electrocardiogram. The cardiac output is a function of a root of the duration of ejection, so the effect of errors is smaller than might be supposed.

When blood regurgitates through the valves, the cardiac output estimated by the ballistocardiogram 
is the whole systolic output, not that part, estimated by the gas methods, which contributes to the circulation. If the amount of the circulation is to be estimated, this is a disadvantage; if one is concerned with the heart's work, it is an advantage over the gas methods. Evidence has been produced that the relation of the heart's work to its size is of fundamental importance in evaluating myocardial function (5).

\section{Relation to results obtained by.other cardiac output methods}

On comparing our normal standards with those obtained by other cardiac output methods, it must be remembered that our results were obtained under slightly different conditions. The average resting cardiac output per sq. meter of body surface for our healthy subjects of both sexes from 20 to 39 years of age was 1.91 liters. For the men only it was 2.05 liters. This compares with an average value of 2.21 liters found in young men by Grollman (6), and 2.23 liters found by the ethyl iodide method in a group of healthy persons from 20 to 39 years of age. In men from 40 to 89 years studied by Lewis ( 7 ) by the acetylene method, the corresponding figure by decades varied only from 2.38 to 2.22 liters. Figure 2 shows that the mean values obtained from the ballistocardiograms tend to be a little lower than these, although they are not significantly different. This is not surprising. In our method the subject lies relaxed on the table; in all the other methods he must breathe through respiratory apparatus and, in some procedures, perform respiratory gymnastics. Such distractions may well prevent the degree of relaxation possible when our method is employed.

The scattering of our data about the means is less than that of Grollman's 50 medical students between 20 and 30 years of age (6) ; somewhat less in most decades than that found by Lewis (7); and considerably less than that found in healthy persons of varying ages by ethyl iodide (5).

When cardiac output is estimated from the ballistocardiogram, experiments on the effect of meals, mild exercise, and drugs, yield results essentially similar to those obtained by other cardiac output methods (9). Also, in the commoner types of disease, our method yields results consistent with the older methods. This consistency increases our confidence in the ballistic method. By its means we have discovered no generalizations concerning cardiac output which could not have been anticipated from earlier work, but to have the information available on large numbers of patients opens new fields of investigation.

\section{Relation to results obtained by other methods of detecting cardiac abnormalities}

As a general rule, when the ballistic form is abnormal, either the physical examination, the orthodiagram, the electrocardiogram, or all of them, demonstrate gross abnormalities also. The discovery of a major change in ballistic form, when all the routine clinical tests were negative, has been very rare; we can cite only 4 cases. Minor ballistic changes, such as flattening of peaks and notching of waves, occurring only during expiration, have appeared a little more frequently when other tests are negative. On the other hand, normal ballistic form and amplitude in the presence of gross cardiac abnormalities are common findings, giving impressive evidence of the ability of the heart muscle to compensate for its disabilities. Abnormally low ballistic amplitude, indicating a subnormal cardiac output, is often found when no cardiac disease can be demonstrated.

\section{Utility of the ballistocardiogram}

Prolonged experience will be necessary before the final value of the ballistocardiogram can be fully assessed. But three promising fields of usefulness can be pointed out.

The detection of circulatory abnormalities. $\mathrm{Pa}-$ tients with subnormal circulations have proved to be common in medical wards. Many have organic heart disease, but a large proportion give no evidence of cardiac abnormality (8). The distinction between disease of the heart and disease of the circulation, justified by physiological reasoning and clinical experiment (5), is now possible routinely. This part of our work will be reported elsewhere.

Changes in single individuals. When the ballistocardiogram is used to assess changes occurring in one individual, most of its uncertainties disappear. Figure 4 shows changes in the circulation induced in normal persons by amyl nitrite and adrenalin, in a case of hyperthyroidism by partial thyroidectomy, in a case of hypertension by thio- 
cyanate, in a case of constrictive pericarditis by pericardiectomy, in a case of hemorrhage by transfusion, and in a case of diabetic acidosis by recovery from the acidosis. Figure 3 illustrates the effect of changes of cardiac rhythm.

The ballistocardiogram provides an easy means of following the course of circulatory and cardiac disease and assessing the influence of treatment upon it. We believe it will be of real value in the investigation of such problems.

The subdivision of existing groups. After acute coronary infarction the ballistocardiograms of some patients give evidence of weakness of one side of the heart; in other cases both sides are equally affected. In some of these patients the cardiac output remains within the normal range; in others it is greatly diminished. After recovery from the acute episode some patients give normal records; others continue to show alarming abnormalities.

Some cases with bundle branch block show weakness of one side of the heart as one would expect; others give perfectly normal ballistic records.

Some cases of hypertension maintain their circulations within the normal range, usually by means of cardiac hypertrophy; in others the circulation is found to be diminished so that the hypertension is maintained without more cardiac work than normal. Some of these cases exhibit ballistic asynchronism as if the left ventricle was showing the first signs of being unequal to the strain; others carry a high hypertension without a trace of this abnormality.

Other groups such as rheumatic heart disease, hyperthyroidism, etc., can be similarly subdivided.

It seems reasonable to expect that information of this kind will increase our understanding of such cases and that this additional insight may lead to improved methods of caring for them.

\section{SUMMARY AND CONCLUSIONS}

Ballistocardiograms, i.e. records of the heart's recoil and the blood's impacts, have been obtained on 300 normal persons and over 400 patients. This method requires nothing of the subject save that he lie on the table. No special training is needed by the operator and the time required is about the same as for an electrocardiogram.

The amplitude of the ballistocardiogram is related to the cardiac output. Normal standards for cardiac output have been defined by tests on 200 healthy persons from 20 to 84 years of age.

The form of the ballistocardiogram is determined by the changes of systolic blood velocity in the great vessels. The normal form has been defined. The common abnormalities have been described, their physiological interpretations set forth, and their clinical significance discussed.

The ballistocardiograph makes possible the routine estimation of the amount of the circulation over most, but not all, of the clinical field. It also provides evidence concerning cardiac health or disease of a type not obtainable by other methods.

This method seems particularly adapted to study the course of diseases of the heart and circulation in single individuals, and to assess the influence of therapeutic agents in such conditions.

\section{BIBLIOGRAPHY}

1. Starr, I., Rawson, A. J., Schroeder, H. A., and Joseph, N. R., Studies on the estimation of cardiac output in man, and of abnormalities in cardiac function, from the heart's recoil and the blood's impacts; the ballistocardiogram. Am. J. Physiol., 1939, 127, 1.

2. Bazett, H. C., Cotton, F. S., Laplace, L. B., and Scott, J. C., The calculation of cardiac output and effective peripheral resistance from blood pressure measurements, with an appendix on the size of the aorta in man. Am. J. Physiol., 1935, 113, 312.

3. Lewis, W. H., Jr., Changes with age in the blood pressures of adult men. Am. J. Physiol., 1938, 122, 491.

4. Davenport, C. B., Body Build and Its Inheritance. Publication 329, Carnegie Institute of Washington, 1923. cf. McLester, J. S., Nutrition and Diet in Health and Disease. W. B. Saunders Co., Philadelphia, 1939.

5. Starr, I., Jr., Donal, J. S., Margolies, A., Shaw, R., Collins, L. H., and Gamble, C. J., Studies of the heart and circulation in disease; estimations of basal cardiac output, metabolism, heart size and blood pressure in 235 subjects. J. Clin. Invest., 1934, 13, 561.

6. Grollman, A., Physiological variations in the cardiac output of man. VI. The valve of the cardiac output of the normal individual in the basal, resting condition. Am. J. Physiol., 1929, 90, 210.

7. Lewis, W. H., Jr., Changes with age in the cardiac output in adult men. Am. J. Physiol., 1938, 121, 517.

8. Starr, I., Tr. A. Am. Physicians, 1939, 54, 163.

9. Starr, I., Gamble, C. J., Margolies, A., Donal, J. S., Jr., Joseph, N., and Eagle, E., A clinical study of the action of 10 commonly used drugs on cardiac output, work, and size; on respiration, on metabolic rate, and on the electrocardiogram. J. Clin. Invest., 1937, 16, 799. 